PROPOSTAS

METODOLÓGICAS

METHODS GUIDELINES

\title{
Revisão e proposta para atualização da diretriz metodológica de análise de impacto orçamentário de tecnologia em saúde para o SUS
}

\author{
Review and proposal to update the methodological guideline \\ for budget impact analysis of health technology for the SUS
}

Márcia Gisele Santos da Costa', Leonardo Castro Luna², Pedro Henrique Amparo da Costa Leite ${ }^{3}$, Bernardo Rangel Tura ${ }^{4}$, Márcia Pinto ${ }^{5}$, Marisa Santos ${ }^{6}$

DOI: $10.21115 /$ JBES.v11.n1.p73-86

\section{Palavras-chave:}

método, impacto orçamentário, Sistema Único de Saúde

\section{Keywords:}

method, budget impact, Unified Health System

\begin{abstract}
RESUMO
Objetivos: Fazer uma proposta para atualização da diretriz com maior padronização dos métodos e realizar análise crítica dos pontos considerados mais relevantes para a formulação de um estudo de análise de impacto orçamentário. Métodos: Revisão da literatura, extração de dados, seleção de etapas básicas para a execução de uma análise de impacto orçamentário, apresentação dos resultados para debate com especialistas. Resultados: Foram recuperados 1.215 títulos, dos quais seis foram elegíveis para síntese qualitativa e definição da estrutura analítica para projetar e construir um modelo de impacto orçamentário: perspectiva da análise; estimar a população-alvo; horizonte temporal; taxa de incorporação da nova tecnologia ao mercado (market share); estimar os custos; fontes de dados; taxa de desconto e apresentação do resultado final. Recomendações: perspectiva da análise deve ser a do pagador; estimativa da população elegível a partir de dados epidemiológicos oriundos do Instituto Brasileiro de Geografia e Estatística (IBGE) preferencialmente; horizonte temporal de dois a cinco anos; buscar a taxa de incorporação em órgãos oficiais; custos diretos estimados a partir da autorização de internação hospitalar e portais de compras governamentais; fontes de dados oficiais e apresentação de resultados em tabela e o impacto incremental ano a ano. Conclusões: Avaliação de Impacto Orçamentário é uma ferramenta feita para auxiliar o gestor, que prima por tentar garantir equilíbrio fiscal ao sistema diante das inúmeras demandas da sociedade. Essa característica é fundamental no mundo atual, onde transparência e a responsabilidade nos gastos são exigências cada vez mais frequentes por parte do cidadão.
\end{abstract}


population; temporal horizon; rate of incorporation of the new technology into the market ("market share"); estimate costs; data sources; discount rate and presentation of the final result. Recommendations: the perspective of the analysis should be that of the payer; the estimation of the eligible population from epidemiological data from the Brazilian Institute of Geography and Statistics (IBGE) preferably; time horizon of 2 to 5 years; rate of incorporation with official data; direct costs estimated from hospital admission authorization and government shopping sites; official data sources and presentation of results in table and incremental impact year by year. Conclusions: Budget Impact Analysis is a tool designed to assist the manager, that trying to guarantee a fiscal balance to the system against the innumerable demands of society. This characteristic is fundamental in today's world where transparency and responsibility in spending are increasingly frequent demands on the part of the citizen.

\section{Introdução}

Uma análise de impacto orçamentário (AIO) estima as mudanças esperadas nas despesas de um sistema de saúde após a adoção de uma nova intervenção. Essa análise pode ser independente ou parte de uma avaliação econômica junto com a análise de custo-efetividade (ACE) (Sullivan et al., 2014). A AlO é uma ferramenta que estima a consequência financeira da incorporação de novas tecnologias a um sistema de saúde específico e pode ser usada como um estudo adicional que fortalece a tomada de decisão para tecnologias que já se mostraram custo-efetivas (Brasil, 2011).

Uma AlO compara cenários, e não tecnologias. O cenário de referência representa a participação no mercado, no momento atual das diversas tecnologias existentes para a condição ou doença estudada (podendo existir várias ou nenhuma tecnologia atual). Os cenários futuros ou alternativos terão uma progressiva incorporação da nova tecnologia proposta, podendo esta modificar não só o uso das tecnologias previamente existentes, como a incidência e desfechos da condição em estudo.

Em setembro de 2012, o Ministério da Saúde publicou seu primeiro manual para a elaboração de análises de impacto orçamentário (Brasil, 2012); e a Portaria SCTIE/MS no 26/2015 reforça a apresentação de estimativa de impacto orçamentário da tecnologia em saúde no Sistema Único de Saúde (SUS) para os primeiros cinco anos de utilização, como um dos requisitos para submissão de proposta de incorporação, alteração ou exclusão de tecnologia em saúde no SUS (Brasil, 2015).

Em um estudo (Niezen et al., 2009), foram levantadas quatro vantagens comparativas da $\mathrm{AlO}$ que justificam a execução desse tipo de avaliação econômica parcial. O cálculo do impacto orçamentário permite quantificar o custo de oportunidade, já que recursos deverão obrigatoriamente ser disponibilizados de outras áreas para a adoção da nova tecnologia. Essa é uma avaliação extremamente importante, só permitida por esse tipo específico de estudo econômico; como existe maior aversão à perda por parte da sociedade em relação à aquisição de ganhos, e por isso maior dificuldade por parte do gestor de propor qualquer desinvestimento, a AlO permite mensurar essas perdas. Outra vantagem é a avaliação da incerteza em características centrais de uma
AIO (como cálculo da população-alvo e custos), por meio de uma análise de sensibilidade bem realizada, favorecendo a antecipação de possíveis déficits financeiros e a identificação das variáveis que mais possam contribuir para esse resultado adverso. Por fim, existe uma preferência pela adoção de tecnologias que possam favorecer toda a população-alvo em detrimento de apenas um subgrupo específico (equidade), mesmo quando exista um custo-efetividade maior em alguma subpopulação. A AlO permite estimar tais orçamentos (população total x subgrupos) e apresentar ao gestor quais as tecnologias são viáveis e para que tamanho de população (Niezen et al., 2009).

O primeiro passo para realizar uma AIO é estabelecer uma estrutura analítica, exemplificada no Quadro 1, que deverá ser adaptada conforme a tecnologia a ser avaliada e que fornecerá uma abordagem geral. Um diagrama de fluxo pode ser útil para ilustrar os parâmetros que serão incluídos na AIO e as categorias de custos que serão estimadas. É necessário ter conhecimento do sistema de saúde da estrutura de saúde implicada no processo de cuidado do paciente, desde a suspeita diagnóstica até, em alguns casos, a reabilitação. É fundamental compreender como a nova tecnologia afetará o orçamento para um plano ou área específica da saúde. Os componentes mais importantes para essa estrutura são a população elegível, o uso potencial da nova tecnologia, a perspectiva de custo do detentor do orçamento e o horizonte temporal (Mauskopf et al., 2017).

Duas revisões sistemáticas de análises de impacto orçamentário para medicamentos consideraram que os estudos possuíam um padrão metodológico inadequado, principalmente em relação a dados importantes como cálculo populacional e custos (van de Vooren et al., 2014; Orlewska \& Gulácsi, 2009). Relatam ainda o enorme predomínio de análises patrocinadas pela indústria farmacêutica na literatura, o que nos leva a inferir que boa parte dos estudos realizados por agências governamentais fica restrita à literatura cinzenta, sem publicação indexada. Concluem sugerindo que pesquisa e investimento no desenvolvimento de melhores métodos para esse tipo de análise econômica são necessários, com o objetivo de assegurar confiabilidade dos dados publicados. Como reforça Neumann (Neumann, 2007), "a procura por padronização e rigor metodológico ajudará na con- 
solidação da AlO" no campo dos estudos econômicos. E sem essa discussão sobre as melhores práticas, continuaremos a basear as decisões de políticas de saúde em evidências de qualidade duvidosa.

A partir dos dados previamente apresentados, pode-se concluir que a AlO é uma ferramenta com crescente demanda por parte dos governos e de central importância para o cálculo do custo de oportunidade na incorporação de novas tecnologias e planejamento das políticas de saúde e seus gastos. Esse é um instrumento ainda em desenvolvimento no mundo todo, com orientações metodológicas em discussão. Daí a necessidade de uma constante avaliação crítica e

Quadro 1. Modelo de diagrama de fluxo para estrutura analítica da AlO

\section{População elegível}

Pacientes no país ou região com diagnóstico da doença que poderão se beneficiar da nova tecnologia.

2. Horizonte temporal

\section{Tratamento atual e futuro com mix de tratamentos}

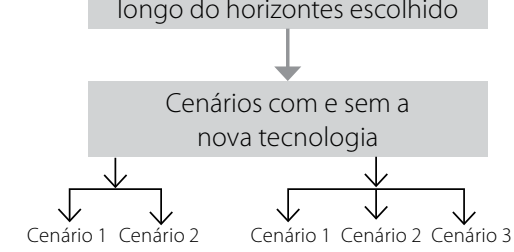

\section{Custos relacionados ao tratamento}

\section{Custos relacionados à condição de saúde}

6. Impactos na saúde da população e no orçamento
População do país (especificada por sexo ou idade, se for o caso)

Prevalência ou incidência da doença

Pacientes em tratamento com tecnologia atual

$\%$ dos pacientes elegíveis para a nova tecnologia

Descrição do impacto anual ao longo do horizontes escolhido
Fonte: Adaptado do diagrama de fluxo de análise de impacto orçamentário do ofatumumabe para tratamento de primeira linha de leucemia linfocítica crônica (Mauskopf et al., 2017, cap. 2, p. 31). proposta de orientações mais objetivas e uniformes que facilitem a formulação desse tipo de estudo econômico no país. O objetivo deste estudo foi fazer uma proposta para atualização da diretriz com maior padronização dos métodos e realizar análise crítica dos pontos considerados mais relevantes para a formulação de um estudo de AIO.

\section{Método}

Este estudo foi concebido em cinco etapas.

\section{Revisão da literatura}

Utilizada para levantamento das práticas atuais e de artigos com críticas ao método utilizado para estudos de $\mathrm{AlO}$, de forma a contribuir para a definição das melhores práticas. Para a execução desta revisão, foram utilizadas cinco estratégias:

a) Busca manual nas referências dos documentos oficiais do Brasil e da International Society for Pharmacoeconomics and Outcomes Research (ISPOR) sobre o tema;

b) Consulta à lista de países que possuem orientações oficiais para análises econômicas (não necessariamente apenas $\mathrm{AIO}$ ) e seus respectivos documentos, disponíveis no site da ISPOR;

c) Contato por e-mail com todas as agências de ATS membros da Rede Internacional de Agências para ATS (The International Network of Agencies for Health Technology Assessment - INAHTA), com objetivo de obter diretrizes ou mesmo recomendações para a elaboração de $\mathrm{AlO}$;

d) Revisão da literatura sobre o tema. Consulta realizada nas bases de dados e no portal da Biblioteca Virtual em Saúde em agosto e dezembro de 2017 (critérios de inclusão: diretrizes oficiais e não oficiais sobre $\mathrm{AlO}$ dos diferentes países, além de artigos críticos da literatura a respeito do tema). Critérios de exclusão: artigos de aplicação do método. Não foram revisadas diretrizes ou recomendações de outros tipos de avaliações econômicas. A busca estruturada encontra-se descrita no Quadro 2.

e) Utilização do livro "Budget-impact analysis of health care interventions - A practical guide" (Mauskopf et al., 2017) para discussão e exemplos.

\section{Extração de dados}

A partir das diretrizes metodológicas localizadas, foram extraídas informações concernentes à estrutura do documento e aquelas relacionados ao método de análise. A partir desses achados, foi elaborada uma planilha com uma síntese qualitativa descrevendo os itens utilizados nos estudos de AlO e suas principais características. 
Quadro 2. Busca estruturada nas bases de dados

\begin{tabular}{lll}
\hline Bases de dados & Estratégia de busca & Artigos recuperados \\
\hline & (("budgets"[MeSHTerms] OR "budgets"[AllFields] OR "budget"[AllFields]) & \\
Medline (via PubMed) & AND ("Impact (Am Coll Physicians)"[Journal] OR "impact"[AllFields]) & \\
& AND ("analysis"[Subheading] OR "analysis"[AllFields])) AND & 1.101 \\
\hline Lilacs & "impamans"[MeSHTerms]) - realizada em 29/12/2017. \\
\hline Biblioteca Virtual em Saúde (BVS) & "realizada em 29/12/2017 & 57 \\
\hline
\end{tabular}

Fonte: Elaboração própria.

\section{Seleção de etapas básicas para execução de uma AlO}

Uma planilha com os resultados obtidos na etapa anterior foi apresentada ao grupo de discussão do Núcleo de Avaliação de Tecnologias em Saúde do Instituto Nacional de Cardiologia (NATS-INC) para a seleção dos componentes básicos para a execução de uma AIO e análise crítica de fontes de dados, suas limitações e melhores práticas à luz do conhecimento atual.

\section{Apresentação dos resultados}

Os resultados preliminares foram apresentados para o fórum de discussão no Ministério da Saúde, com membros do Departamento de Gestão e Incorporação de Tecnologias em Saúde (DGITS) e do Departamento de Economia da Saúde, Investimentos e Desenvolvimento (DESID), no qual cada etapa básica para a realização de uma AIO foi debatida amplamente com relação a sua inserção na análise, e opções de fontes de dados.

\section{Considerações éticas}

Potencial conflito de interesses por parte dos autores: nenhum.

Este projeto foi submetido à Plataforma Brasil e aprovado em 10/02/2015 pelo Comitê de Ética do Instituto Nacional de Cardiologia sob o nº de identificação 41220915.6.0000.527.

\section{Resultados}

Os documentos oficiais do Brasil (Brasil, 2012) e da ISPOR sobre AIO citam outras diretrizes internacionais: a versão brasileira nos referencia aos documentos do Canadá (Marshall et al., 2008), Reino Unido (Nice, 2011), Austrália (PBAC, 2013) e Polônia (Poland, 2009), enquanto a versão da ISPOR relaciona uma lista de países que possuem diretrizes em avaliação econômica (Sullivan, 2014).

Do site da ISPOR na seção "Guidelines around the world" (ISPOR), foram incluídos quatro novos documentos: da Irlanda (Ireland, 2010), Bélgica (Thiry et al., 2014), França (Boulenger \& Ulmann, 2004) e Malásia (Malaysia, 2012).

Das cerca de 50 agências de ATS, de 34 países, membros do INAHTA, apenas seis responderam ao e-mail (Colômbia,
Malásia, Alemanha, Espanha, Áustria e Suécia) e apenas duas encaminharam documentos: uma diretriz colombiana não oficial e um documento austríaco em alemão (este último foi excluído por causa do idioma).

Na revisão da literatura, tendo como critério de inclusão a possibilidade de contribuição crítica de cada artigo sobre a formulação metodológica de uma AlO, a partir da busca no Medline, foram recuperados 1.101 títulos; separaram-se 38 artigos para a leitura dos respectivos resumos, sendo selecionados 27 artigos para leitura completa (desses, não tivemos acesso a dois documentos completos tailandeses). Na base Lilacs, foram localizados 57 títulos, sendo selecionados dois artigos para leitura dos respectivos resumos e um artigo para leitura completa; e na BVS, retornaram 57 títulos da busca e os quatro artigos selecionados para leitura do resumo não foram elegíveis. Ao final, foram utilizados seis artigos para discussão (Figura 1). Os artigos selecionados encontram-se resumidos no Quadro 3.

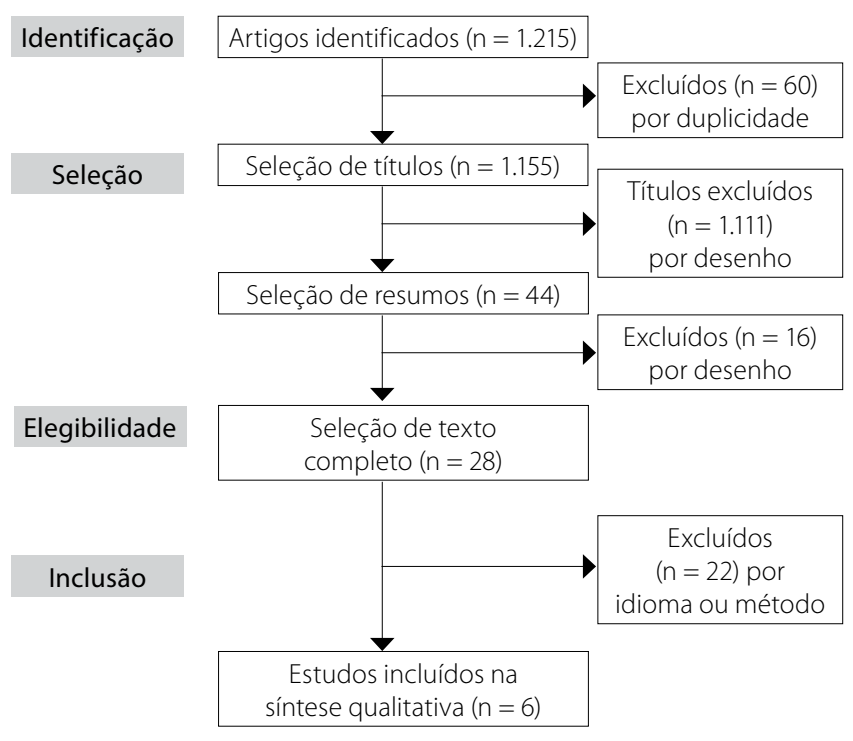

n = número de artigos

Fonte: Adaptada do Prisma Flow Diagram (Moher et al., 2009)

Figura 1. Fluxograma da revisão da literatura. 
Quadro 3. Resumo dos artigos críticos sobre metodologia de AIO selecionados nas bases de dados

\begin{tabular}{|c|c|}
\hline Artigo & Comentários \\
\hline Trueman et al. (2001) & $\begin{array}{l}\text { Aborda o que é necessário para uma AIO. Ressalta que fazer uma análise na perspectiva da sociedade, } \\
\text { tendo como desfecho redução nas perdas por produtividade, não é relevante para o decisor, uma vez } \\
\text { que o recurso economizado não é repassado ao setor da saúde. Os autores destacam as seguintes } \\
\text { qualidades numa AIO: transparência (compreensão clara de todas as entradas; na existência de um } \\
\text { modelo preditivo, este deverá ser interativo, possibilitando adaptações às práticas locais); perspectiva } \\
\text { (preferencialmente do pagador; tem que ficar bem clara, pois estará diretamente relacionada à } \\
\text { relevância dos custos e benefícios); fontes de dados confiáveis (claramente indicada juntamente com } \\
\text { quaisquer pressupostos adicionais feitos na ausência de dados); taxas de adoção da nova tecnologia } \\
\text { (importante considerar substituição do tratamento existente e a demanda induzida); impacto da } \\
\text { intervenção por subgrupos da população ou indicações de forma a racionalizar a disponibilidade } \\
\text { do tratamento; apresentação de resultados (o impacto do serviço deverá ser relatado em unidades } \\
\text { naturais (por exemplo, consultas); relocação de capital humano e financeiro; horizonte temporal } \\
\text { (período que satisfaça o demandante e que será influenciado pela terapia sob observação, a natureza } \\
\text { dos benefícios acumulados e da taxa de adoção); análise de sensibilidade (devido a grandes incertezas } \\
\text { nos parâmetros); participação do decisor no modelo (discussão sobre estrutura e entradas). }\end{array}$ \\
\hline $\begin{array}{l}\text { Orlewska \& } \\
\text { Mierzejewski (2004) }\end{array}$ & $\begin{array}{l}\text { O objetivo do artigo foi apresentar o projeto para diretriz de AlO da Polônia. Principais recomendações: } \\
\text { transparência e fácil entendimento de todos os pressupostos e dados utilizados na análise e da } \\
\text { relação entre as variáveis e os desfechos; fonte de dados específica do país, seleção de fontes de } \\
\text { custos consistentes com a perspectiva da análise e dados de efeito da tecnologia (p. ex.: mortalidade, } \\
\text { eventos adversos) podem ser obtidos de fontes internacionais, devem ser coletados por meio de } \\
\text { revisão sistemática ou medicina baseada em evidências e, se possível, adaptados para o país, e a } \\
\text { seleção da fonte deve ser justificada. Perspectiva do financiador público. Horizonte temporal: até que } \\
\text { o produto atinja uma participação máxima ou estável no mercado ou com pelo menos } 2 \text { anos de } \\
\text { incorporação. População ou subgrupos definidos com base na indicação e nas possíveis diferenças } \\
\text { de eficácia, custos ou preferências. Os resultados devem ser apresentados em unidades monetárias e } \\
\text { naturais, e impactos no serviço. Modelo deve prever relocação de recursos e prever como as economias } \\
\text { serão realizadas no tempo. Inclui análise de sensibilidade e informações sobre a tecnologia. }\end{array}$ \\
\hline Orlewska \& Gulácsi (2009) & $\begin{array}{l}\text { Os autores avaliaram a qualidade dos estudos de AIO tendo como referência as } \\
\text { recomendações da ISPOR. Nessa revisão da literatura eles incluíram } 34 \text { estudos, no } \\
\text { período de pesquisa de } 2000 \text { a 2008. Observaram que os estudos são falhos na mudança } \\
\text { da população ao longo dos anos; não fornece informações no formato correto (valor do } \\
\text { impacto tanto total como incremental desagregado ao longo do tempo e calculado para } \\
\text { a população completa de interesse) e na apresentação de análises de sensibilidade. }\end{array}$ \\
\hline
\end{tabular}

Descrevem o desenvolvimento de um modelo genérico projetado em uma planilha de cálculo que pode ser aplicada a qualquer tecnologia em saúde e que possibilita obter estimativas com diferentes graus de complexidade. Os autores identificam os componentes determinantes de uma $\mathrm{AIO}$ como sendo: população (extrapolação da população a partir de registros de casos, dados da literatura, ou ainda da população geral que pode ser estruturada por sexo e idade; a seguir, aplicamse taxas de prevalência e incidência da literatura validadas por consenso de experts como painel

Garay et al. (2011) Delphi. Ideal que se façam ajustes levando-se em consideração a aderência ao tratamento, se doenças crônicas ou agudas (ajuste temporal), crescimento populacional, e outros ajustes) custos (diretos, de componentes associados ao uso, coberturas e eventos adversos, pode se usar taxas como inflação ou não), horizonte temporal (suficiente para incorporar todos os determinantes do modelo e com base nas necessidades do pagador), taxa de difusão (Market share - determinada pela instituição, decisões políticas ou mesmo por características da própria tecnologia), que a perspectiva da análise seja a do pagador, e análise de sensibilidade (variar parâmetros de maior impacto).

Revisão da literatura sobre métodos de AIO no período de 2001 a 2011, sendo selecionados 5 artigos, com objetivo de melhor esclarecer o papel da $\mathrm{AIO}$ em relação às outras avaliações econômicas.

Garattini et al. (2011) Os autores sugerem que a AIO seja na perspectiva do detentor do orçamento; comparador seja o tratamento atual; horizonte temporal menor que 3 anos; não usar taxa de desconto; incluir os custos diretos, resultados expressos em custo incremental e análise de sensibilidade. A novidade foi como incluir a efetividade nos resultados como potenciais economias a curto prazo.

Revisão sistemática crítica das AIO de países da Europa de 2008 a 2014. Os critérios para avaliar a qualidade dos estudos foram: perspectiva do detentor do orçamento; curto horizonte temporal; se era um novo tratamento; custos em moeda local; eficácia a curto prazo; custo incremental na população-alvo; análise de sensibilidade simplificada; variação do mercado. A conclusão dos autores foi de que a AIO ainda não estava bem estabelecida, com estudos de baixa qualidade, talvez pela lacuna de informações da prática real que é necessária para esse tipo de avaliação.

Fonte: Elaboração própria. 
Após a leitura das diretrizes localizadas, foram extraídos dados referentes ao documento, e seu conteúdo foi resumido no Quadro 4. A partir desses dados, foram definidas pelo grupo de discussão do NATS-INC a estrutura analítica para projetar e construir um modelo de impacto orçamentário: perspectiva da análise; estimativa da população-alvo; horizonte temporal; taxa de incorporação da nova tecnologia ao mercado (market share); estimativa dos custos; fontes de dados; taxa de desconto e apresentação do resultado final.

\section{Discussão}

\section{Perspectiva da análise}

Em relação à perspectiva, há pequenas variações devido à heterogeneidade dos diversos sistemas de saúde envolvidos. Existe o consenso geral de que as AlO são estudos estritamente financeiros e de interesse direto do detentor do orçamento. Na medida em que são produzidas com o objetivo de ajudar a definir o seu orçamento contábil, devem refletir a perspectiva de sua visão direta. Alguns documentos citam situações excepcionais nas quais o contratante é uma entidade para a qual outros aspectos indiretos como benefícios previdenciários ou trabalhistas são importantes (NICE, 2011; Orlewska \& Mierzejewski, 2004; Cohen et al., 2008). A perspectiva adotada em uma AIO deve ser claramente estabelecida, e os custos e economias, considerados consistentes com essa abordagem (Trueman et al., 2001). Com o grupo de discussão na Comissão Nacional de Incorporação de Tecnologias no SUS (Conitec), acordou-se que a perspectiva deve ser a do pagador, por exemplo, o Ministério da Saúde, Secretarias Estaduais/Municipais ou Hospitais.

\section{População-alvo}

A definição da população com indicação de uso da nova tecnologia deverá especificar se existem pacientes de alguma subpopulação de interesse especial (que teria um benefício maior) e se há restrição para a elegibilidade. Deve-se relatar se a nova tecnologia impactará o tamanho da população ou a condição de saúde (mudanças nos níveis de gravidade, maior sobrevida ou redução da transmissão de infecções, por exemplo) ao longo do tempo (Mauskopf et al., 2017).

Para o cálculo da população de uma AlO, existe uma preferência geral para que ela seja calculada por métodos epidemiológicos, notadamente a prevalência para doenças crônicas e a incidência para doenças agudas. A diretriz brasileira é abrangente neste tópico, sugerindo como fonte de dados preferencialmente agências oficiais (apesar de não as citar), caracterizando a população de estudo como aberta e levando em consideração as restrições de indicação oficial para o uso da nova tecnologia. Erro no cálculo do tamanho da população pode gerar consequências catastróficas, por outro lado, a incorporação de uma tecnologia pode gerar economias e externalidades. O cálculo do tamanho da população por método epidemiológico pressupõe um acesso "ideal" ao sistema de saúde, muitas vezes limitado por questões regionais ou de vulnerabilidade das populações-alvo.

A ISPOR sugere considerar o uso futuro fora das restrições impostas pelo governo (chamado "leakage"), o que a diretriz brasileira chama de "demanda forçada". Esses casos são representados por populações que apresentam indicação formal para uso da nova tecnologia, mas que, por restrição orçamentária ou priorização de subgrupos com melhor relação de custo-efetividade, têm o acesso limitado ou negado a ela. O documento australiano prevê o cálculo da população por demanda aferida quando a nova tecnologia for não inferior à atual. E as demais diretrizes oficiais pouco acrescentam a essas orientações. Deve-se desestimular a inclusão do uso da nova tecnologia além da indicação para qual foi aprovada no ato de registro na Anvisa (no caso de medicamentos, o uso além da indicação descrita na bula - off-label), que poderia influenciar a prescrição para situações clínicas sem a devida evidência científica de eficácia ou segurança, principalmente em realidades como a brasileira, onde já existe importante judicialização da saúde.

John Watkins e Dan Danielson (Watkins \& Danielson, 2014), escrevendo sob a ótica das empresas privadas de saúde americanas, advogam que o uso off-label seja mensurado e descrito na AlO. Isso deve acontecer quando esse uso for julgado importante ou houver no presente momento outras potenciais indicações em desenvolvimento para essa tecnologia sob análise - o que envolve monitoramento do horizonte tecnológico. Isso pode refletir uma prática já existente ou futura do mercado que extrapole as recomendações oficiais, mas que talvez influencie de maneira significativa o cálculo de custo final para o sistema de saúde (Watkins \& Danielson, 2014). Orlewska e Mierzejewski (Moher et al., 2009), em orientações polonesas sobre impacto orçamentário, também sugerem que a mensuração do uso off-label pode ajudar a produzir previsões futuras de mercado mais realistas e ajudar na elaboração de medidas preventivas para essas extrapolações de prescrição. Cohen et al. (2008) ainda lembram a possibilidade de expansão do mercado por pacientes não tratados que, a partir da incorporação da nova tecnologia, podem também demandar por ele. O método de demanda aferida para o cálculo da população ficaria exclusivo apenas para análises nas quais o gestor definisse esse tipo de metodologia como de sua preferência e disponibilizasse os dados populacionais de interesse. Garay et al. (2011) sugerem ainda um "ajuste" a mais para a população-alvo, por meio da estimativa, por parte dos profissionais de saúde, da provável aderência dos pacientes à prescrição da nova tecnologia. Por ser uma avaliação na maioria das vezes subjetiva, baseada na experiência de cada profissional, talvez esse dado agregue mais incerteza ao cálculo final do que informação relevante. 


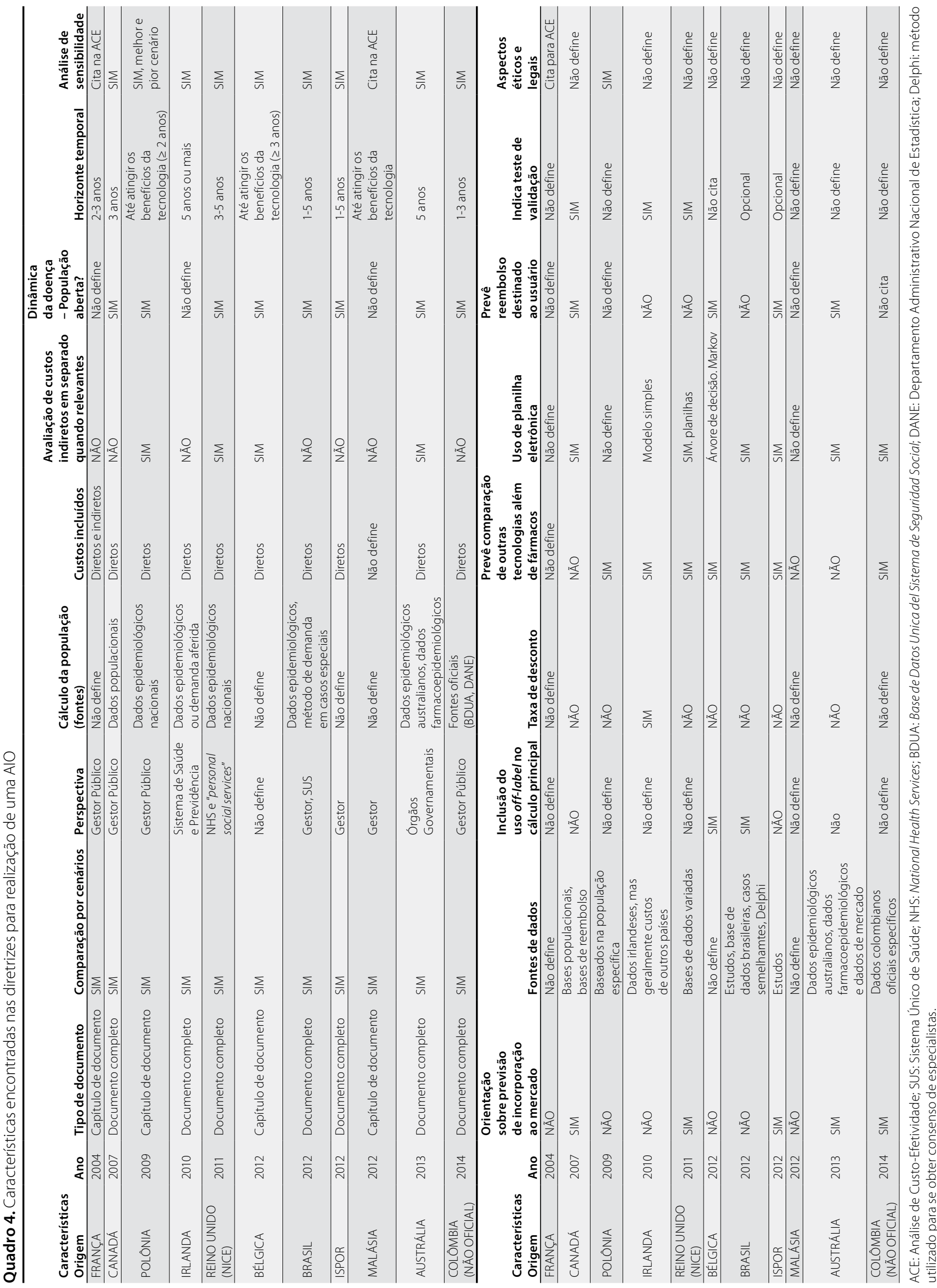


O grupo de discussão no Ministério da Saúde concluiu que o uso além da indicação descrita em bula (off-label) não deverá ser considerado nos cálculos, mas as estimativas de demanda forçada (leakage), isto é, que extrapolem as restrições de uso, sim. Essas estimativas não possuem fonte de dados e podem ser obtidas por meio de pesquisas de mercado ou opinião de especialistas. Os pressupostos adotados e o método utilizado para estimar o percentual de pacientes oriundos da demanda forçada deverão ser descritos detalhadamente. A população deverá ser estimada para cada ano da análise contemplado no horizonte temporal, com e sem a nova tecnologia.

A estimativa da população elegível deverá ser realizada a partir de dados epidemiológicos oriundos de informações disponibilizadas pelo Instituto Brasileiro de Geografia e Estatística (IBGE) - projeção da população do Brasil; a PNAD (Pesquisa Nacional por Amostra de Domicílio); a PNS (Pesquisa Nacional de Saúde); ou ainda a Vigitel (Vigilância de Fatores de Risco e Proteção para Doenças Crônicas por Inquérito Telefônico), nessa ordem de preferência, e a escolha da fonte deverá ser justificada. Quando o dado populacional for de outra natureza (por exemplo, documentos de sociedades médicas ou da indústria farmacêutica), deve-se exigir que o autor confira se ele se baseia nos dados governamentais e deixe essa informação bem-definida, além de justificar o não uso de uma fonte oficial. Muitas vezes é preciso o acesso a outras fontes de dados para complementar as informações necessárias à elaboração do modelo.

Para chegar a uma estimativa do tamanho da população elegível, recomenda-se um processo de "afunilamento" da população total para aqueles que são elegíveis para a nova tecnologia. Essa abordagem pode ser realizada por meio de uma série de cálculos usando dados como: tamanho total da população; distribuição da idade e do sexo na população (dependendo da condição de saúde objeto da análise), se for o caso; incidência anual (por idade ou sexo, se for o caso) para uma condição aguda, ou incidência e prevalência para uma condição crônica, uma vez que a população é aberta, ocorrendo entradas (novos casos) e saídas (por melhora ou óbito, por exemplo) ao longo do período; porcentagem da população incidente ou prevalente com diagnóstico; porcentagem da população incidente ou prevalente com diagnóstico e com indicação para a nova tecnologia (população elegível).

\section{Horizonte temporal}

O horizonte temporal na maioria das diretrizes prevê a projeção orçamentária para um futuro de pequeno a médio prazo, entre um e cinco anos. A exceção é o documento polonês, que sugere um tempo maior que dois anos e que seja suficiente para se atingir a resposta terapêutica esperada da nova tecnologia proposta. Idealmente, o horizonte temporal de uma AIO não deve ser preestabelecido de maneira fixa, mas sim delineado em cada estudo de acordo com a perspectiva de incorporação da nova tecnologia até que essa atinja seu estado de equilíbrio no mercado ou que proporcione parte relevante de seu impacto sobre o setor de saúde. Assim, no caso da incorporação de tecnologias que já sejam de conhecimento prévio dos profissionais e de ampla distribuição no mercado, possivelmente um horizonte temporal curto (de um a dois anos) será suficiente. Ao contrário, em situações em que haja a necessidade de obras de implementação, compra de maquinário, desenvolvimento de diretrizes para a prática clínica, curva de aprendizagem, desconhecimento por parte dos profissionais e outros obstáculos que dificultem a absorção e a plena eficiência da tecnologia proposta, horizontes temporais mais longos serão necessários. Outros estudos (Garanttini \& van de Vooren, 2011; van de Vooren et al., 2014; Trueman et al., 2001) enfatizam que horizontes temporais mais longos levantam a questão de se investir em uma tecnologia no presente que talvez só traga vantagem financeira no futuro. Do ponto de vista do gestor, existirá a necessidade atual de gerar receitas adicionais ou impor um desinvestimento em outra área da saúde para financiar essa nova tecnologia, o que muitas vezes pode não ser politicamente exequível no mundo real e resultar em redução de saúde para a sociedade no curto prazo. A dinâmica da doença ou da própria tecnologia pode variar em um curto espaço de tempo sendo necessário refazer a AlO.

A conclusão do grupo de discussão foi de que o horizonte temporal poderá variar de dois a cinco anos, tendo em vista que algumas tecnologias, ainda não incorporadas pelo SUS, mas consolidadas no mercado, teriam uma taxa de adoção elevada no primeiro e no segundo ano, correspondendo a um período mínimo de estabilização da tecnologia incorporada, como relatado por Orlewska \& Mierzejewski (2004).

\section{Taxa de incorporação (market share)}

Em relação à taxa de incorporação de uma nova tecnologia ao mercado, ou market share, pouca orientação objetiva existe em quase todos os documentos oficiais. A diretriz brasileira (Brasil, 2012) traz mínimas sugestões: a extrapolação a partir da curva de incorporação de outra tecnologia semelhante, no sistema de saúde brasileiro; a extrapolação a partir da curva de incorporação da mesma tecnologia em outro mercado; ou ainda um painel de especialistas (painel Delphi). A ISPOR comenta a dificuldade dessa previsão e a importância de testar diferentes estimativas na análise de sensibilidade dessa variável. Nesse tópico, a diretriz canadense (Marshall et al., 2008) é a mais extensa e faz uma série de recomendações objetivas e pertinentes: relata que a estimativa de crescimento do mercado depende do crescimento da população e da dinâmica da doença influenciada pela nova tecnologia; se não houver informações sobre esse crescimento populacio- 
nal, deve-se estimá-lo em 0\%; podem ser utilizados dados históricos de tecnologias similares ou de outros mercados já incorporados; sempre incluir comentários sobre a escolha das fontes de dados nas quais foram baseadas as previsões do mercado; usar bases de dados e previsões oficiais sempre que possível para determinar distribuição do mercado de referência e o crescimento da população; avaliar outras tecnologias que possam vir a ser incorporadas ou descontinuadas desse mercado durante o horizonte temporal dessa AlO - essas mudanças podem influenciar a taxa de incorporação da tecnologia estudada; recomenda-se que se citem dados históricos da incorporação em outros mercados; dados da incorporação dessa tecnologia ou de tecnologias similares em outros mercados ou de sistemas privados só devem ser incluídos como fonte de dados se forem semelhantes ao mercado estudado ou se essa relação entre os mercados for bem entendida; se não houver essas previsões, usar dados publicados, opinião de especialistas ou alguma experiência prévia. Um parâmetro importante é estimar o conhecimento dos usuários e dos profissionais de saúde sobre essa nova tecnologia proposta para delinear as previsões; se não houver dados para prever os novos cenários, recomenda-se pelo menos manter nos cenários futuros a proporção das tecnologias já existentes que compõe o cenário de referência.

Ao longo do tempo, as cotas de mercado de uma tecnologia mudam, tanto com a inserção ou não da nova tecnologia no tratamento como por mudanças no padrão de cuidados, com a crescente aceitação de tecnologias recém-aprovadas ou a substituição ou adição aos esquemas de tratamento tradicionais. Para evitar um número excessivo de tecnologias, deve-se incluir na $\mathrm{AlO}$ apenas aqueles produtos utilizados como tratamento-padrão (ou sem tratamento) cujo uso pode ser afetado pela adição da nova tecnologia (Mauskopf et al., 2017).

Não há fontes padronizadas para a estimativa de mudanças no market share de tratamentos. Essas estimativas podem ser feitas pelo fabricante ou pelos pesquisadores após consulta aos especialistas com base nos padrões de tratamento atuais. Um fator que deve ser considerado é que, se outras novas tecnologias tiverem sido recentemente incorporadas, sua participação pode aumentar ao longo do tempo, e até mesmo retirar do mercado o tratamento mais antigo, possivelmente menos eficaz. Se os padrões de tratamento não mudaram recentemente, então se espera que as participações no mercado permaneçam constantes (Mauskopf et al., 2017). Um segundo fator a se considerar no caso de medicamentos é se novos medicamentos de marca deverão ser lançados dentro do horizonte temporal de análise, e um terceiro fator seria a comercialização de genéricos, que nesse caso poderia aumentar a disponibilidade do medicamento (marca mais genérico) e consequentemente sua participação no mercado (Mauskopf et al., 2017). É importante que os pressu- postos feitos sobre mudanças que poderão ocorrer nas cotas de mercado ao longo do horizonte temporal sejam descritos e que essas estimativas estejam contempladas na análise de sensibilidade. Com a entrada de uma nova tecnologia de marca ou medicamento genérico durante o horizonte temporal, além de estimar o impacto nas cotas de mercado, os desenvolvedores da análise precisam considerar o custo do tratamento e o impacto potencial sobre os resultados clínicos na população total elegível, caso sejam relevantes (Mauskopf et al., 2017).

Como opinião dos autores e em concordância com a diretriz canadense, definir a atual familiaridade e extensão do uso corrente da nova tecnologia por parte dos profissionais de saúde tem grande influência na velocidade de incorporação dela. Por exemplo, se um medicamento estudado já é amplamente utilizado nos serviços privados de saúde e faz parte de recomendações oficiais de sociedades médicas, sua incorporação após aprovação no sistema público provavelmente será bastante acelerada. Da mesma maneira que o investimento em propaganda por parte do fabricante também influenciará nas projeções de incorporação da nova tecnologia. Existem tabelas oficiais de projeção para o crescimento da população brasileira elaboradas pelo IBGE, assim como o pesquisador deve buscar na indústria farmacêutica ou nos órgãos oficiais estimativas de distribuição do mercado para as tecnologias do cenário atual. Outra estratégia para estabelecer a distribuição do mercado atual, que será a base inicial para a previsão de incorporação da nova tecnologia nos mercados futuros ou alternativos, é a quantificação das vendas em período prévio dos principais produtos concorrentes nas bases de compras oficiais já citadas. Recomenda-se fazer 0 levantamento das compras realizadas para produtos concorrentes num período prévio de 12 meses e determinar o percentual correspondente a cada produto, que será utilizado como estimativa de cota de mercado.

\section{Custos}

Os custos que devem ser levados em consideração para o cálculo do impacto orçamentário de uma nova tecnologia são somente os diretos médicos. Esse é um consenso entre as diretrizes oficiais e incluem o valor da nova tecnologia específica, dos exames diagnósticos necessários, do manejo de seus efeitos colaterais e dos principais desfechos clínicos (por exemplo, hospitalizações), de sua implementação e capacitação, da confecção de novas diretrizes de práticas clínicas etc. Custos indiretos, como os previdenciários ou com os tratamentos de outras patologias futuras por aumento de sobrevida e cura, não devem entrar nesse tipo de análise econômica. Os documentos da Austrália (PBAC, 2013) e da Polônia (Poland, 2009) sugerem que impactos indiretos relevantes sejam citados à parte, sem que façam parte do cálculo principal. Como a diretriz irlandesa também aborda tecnologias 
como equipamentos, ela prevê que se inclua custo capital (por exemplo, edifícios, reformas, terrenos e outros) e sugere o uso de uma taxa de depreciação nesse tipo específico de situação. Alguns tipos de custos são considerados não recuperáveis (sunk costs), por exemplo, edificações. Garay et al. (2011) chamam ainda a atenção para o cálculo do percentual de desperdício que possa acontecer, por exemplo, pelo descarte de alguns miligramas da dose de um medicamento em ampola, ou de alguns comprimidos de uma caixa com um número superior ao necessário. Afirma que, se a perda estimada for importante, ela poderá impactar de maneira significativa no cálculo final.

No Brasil, existem fontes oficiais e gratuitas de custos que devem ser idealmente descritas na diretriz nacional e seu uso estimulado. O Portal de Compras do Governo Federal (Compras Governamentais) tem o registro do preço praticado nos pregões eletrônicos das compras de todo tipo de produto, das instituições públicas federais, e disponibiliza um link para o Painel de Preços que foi desenvolvido pela Secretaria de Gestão do Ministério do Planejamento, Desenvolvimento e Gestão a partir das compras públicas homologadas no Sistema Integrado de Administração de Serviços Gerais (SIASG) e Compras Governamentais (antigo Comprasnet). No caso de medicamentos que ainda não foram adquiridos pelo SUS, pode ser consultada a Câmara de Regulação do Mercado de Medicamentos (CMED), órgão interministerial responsável por regular o mercado e estabelecer critérios para a definição e o ajuste de preços. Aqui temos uma listagem por ordem alfabética e por diferentes alíquotas de ICMS cobrado pelos estados. Aos preços do CMED, para determinados medicamentos, o governo ainda impõe um Coeficiente de Adequação de Preços (CAP), que é um desconto mínimo obrigatório a ser aplicado sempre que forem realizadas vendas desses medicamentos destinadas aos entes da administração pública direta e indireta da União. Mas, na prática, os preços sugeridos nas tabelas do CMED, mesmo quando aplicado o CAP, ainda ficam muito acima dos praticados pelo mercado, especialmente quando o medicamento já possui apresentações similares e genéricas, sendo recomendado que essa fonte só seja utilizada quando não se tem nenhuma compra pública registrada. O Banco de Preços em Saúde (BPS) é um sistema criado pelo Ministério da Saúde com o objetivo de registrar e disponibilizar as informações das compras públicas e privadas de medicamentos e produtos para a saúde, constituindo outra opção gratuita de consulta a preços de medicamentos praticados no Brasil. Ele depende da disponibilização das informações de maneira voluntária por parte das instituições de saúde, mas, mesmo assim, apresenta um grande número de registros para consulta. A grande vantagem do BPS é apresentar uma média ponderada de preços do produto de interesse para o período buscado. Em relação à busca por custos de procedimentos, a consulta deve ser realizada no
Sistema de Gerenciamento da Tabela de Procedimentos, Medicamentos e Órteses, Próteses e Materiais Especiais (SIGTAP) do SUS. Ainda, pode-se recorrer às Secretarias de Saúde e aos Institutos Nacionais ligados ao Ministério da Saúde como fontes de dados públicos.

A recomendação do grupo de discussão acompanha as orientações internacionais para elaboração da AlO considerar apenas os custos diretos médicos relacionados à tecnologia: preço de aquisição, administração ou utilização (considerar dose, posologia e duração do tratamento no cálculo total), testes diagnósticos (cuidado ao incorporar os custos de diagnóstico nos cálculos do orçamento para uma condição crônica, pois esses custos só seriam válidos para a população incidente, porque eles só foram incorridos uma vez antes de se iniciar um tratamento), monitoramento (por exemplo, dosagem da razão normalizada internacional - RNI [do inglês international normalized ratio - INR] dos pacientes em uso de varfarina) e tratamento de eventos adversos. Utilizar como fonte de preços, nesta ordem de preferência, os valores médios da Autorização de Internação Hospitalar (AlH), o SIGTAP para procedimentos e, para materiais e medicamentos, o portal de compras do Governo Federal (Compras Governamentais), adotando-se o valor unitário da última compra; BPS que fornece a média ponderada do preço unitário dos últimos 18 meses; e no caso de não haver compra registrada, a tabela da CMED para compras públicas. Cabe ressaltar que a tabela SIGTAP corresponde à parcela de investimentos federais, e não ao valor real do procedimento, que, em muitos casos, contém investimentos municipais e estaduais. Importante destacar que esses valores de custos devem corresponder ao ano em que a análise está sendo realizada. No caso de só haver disponível valores de anos anteriores, a atualização desses custos deverá ser feita utilizando-se o valor do Índice Nacional de Preços ao Consumidor Amplo (IPCA) saúde (valor médio dos últimos 12 meses). No caso de produtos importados, com custos em outra moeda, além da conversão, deve-se atentar para situações de instabilidade econômica com variações cambiais em curto prazo (que deverão estar contempladas na análise de sensibilidade).

O grupo concluiu que a estimativa de economia oriunda de potenciais benefícios, como, por exemplo, a redução de internações em unidade de terapia intensiva (UTI), seja calculada à parte, não sendo inserida no cálculo da $\mathrm{AIO}$, uma vez que, na prática, esses recursos acabam sendo utilizados por outros pacientes, não ocorrendo economias reais no orçamento, e sim uma ampliação de acesso.

\section{Fontes de dados}

Em todos os documentos, as fontes de dados geralmente são um assunto não aprofundado. Essas são de extrema importância para definir o cálculo da população estudada e suas projeções de crescimento, para as estimativas de cus- 
to, para a confecção da distribuição do cenário de referência e a previsão das taxas de incorporação ao longo dos cenários futuros. As fontes de cada país ou localidade devem ser oficiais e preferencialmente explicitadas e referenciadas em cada diretriz nacional de estudos econômicos, como forma de orientação uniforme aos pesquisadores. Assim o fazem alguns países, como o Reino Unido (NICE) e a Colômbia.

No Brasil, a diretriz deve recomendar o uso das fontes já citadas (AlH, IBGE, Datasus, Vigitel, PNAD, CMED, BPS, Compras Governamentais e SIGTAP). No caso de uso de fontes alternativas e não oficiais, como documentos de sociedades médicas ou da indústria, o autor deve checar a fonte utilizada e certificar-se de que esse dado seja citado objetivamente. Aqui a transparência deve ser a regra, com o autor sempre deixando claro e justificando todas as suas escolhas.

\section{Taxa de desconto}

A taxa de desconto é o ajuste feito a valores futuros, convertendo-os para o presente por meio da aplicação de uma taxa predeterminada para isso. Esse conceito se baseia na premissa da Economia na qual a sociedade tem a preferência pelo dinheiro hoje, mais do que no futuro, e tem a preferência por contas a pagar no futuro, e não no presente. Como a AIO trabalha com um horizonte temporal geralmente muito mais curto do que a ACE, as diretrizes de estudos econômicos sugerem que não se aplique a taxa de desconto na AIO. A exceção é o documento oficial da Irlanda (Ireland, 2010), que afirma que em certas circunstâncias seria apropriado aplicar tal taxa de desconto em uma AlO, com o objetivo de refletir valores mais verdadeiros que incidiriam no sistema de saúde atual. Não há a exemplificação dessas "situações excepcionais". Em uma AIO, os custos de interesse para o detentor do orçamento são tipicamente os custos contábeis não descontados, que se espera que sejam incluídos no orçamento (Mauskopf et al., 2017).

O grupo de discussão concluiu que esse tipo de ajuste financeiro não se faz adequado para uma AIO.

\section{Apresentação dos resultados}

A tomada de decisão baseada em argumentos acadêmicos e econômicos deve respeitar os tempos, habilidades e recursos do gestor de saúde. Deve-se entender que muitas vezes esses tomadores de decisão não possuem profundo conhecimento de aspectos técnicos em saúde ou economia e, portanto, o resultado final deve ser apresentado de forma simples, de fácil entendimento, e pode ser disponibilizado em uma enorme variedade de formatos: modelos, relatórios, planilhas de cálculo, publicações, entre outros (Mauskopf et al., 2017). Independentemente do formato de apresentação, o relatório da $\mathrm{AlO}$ deve seguir os moldes gerais do Relatório Consolidado de Avaliação Econômica da Saúde Padronizado (CHEERS) para avaliações econômicas (Husereau, 2013). Esses padrões sugerem que uma avaliação econômica seja apresentada com detalhes suficientes para que um pesquisador interessado possa reproduzir os resultados da análise ao usar a estrutura do modelo, os pressupostos e os valores de entrada fornecidos. Para atingir esse nível de transparência, os seguintes elementos devem ser incluídos em todos os diferentes formatos de apresentação citados acima: objetivo da análise; descrição da estrutura analítica e diagrama de fluxo; lista de todos os pressupostos estruturais feitos dentro da análise; valores e fontes de dados ou racional para pressupostos para todos os valores de entrada utilizados para derivar o tamanho da população elegível, market share, desfechos de segurança e eficácia, e custos relacionados à tecnologia e à doença (unitário e total para população elegível). Também devem ser apresentados os resultados do caso base em tabelas para o mix de tratamento com e sem a nova tecnologia e a diferença entre eles (impacto incremental) ano a ano; análises de sensibilidade; validação da análise; resumo dos achados e conclusões; forças e limitações da análise.

A AlO pode conter a apresentação de resultados com diversos cenários, por exemplo, a utilização restrita em centros de referência ou em todo o SUS.

\section{Validação do estudo}

A validação tem por objetivo dar confiança e credibilidade aos resultados oriundos do modelo. Para que um modelo seja útil aos tomadores de decisão além da confiança nos resultados, eles precisam saber com quanta precisão o modelo prediz os resultados de interesse (Eddy et al., 2012). Para isso, deve-se assegurar a transparência (descrevendo claramente a estrutura do modelo, equações, valores dos parâmetros e premissas adotadas) e a validação (submetendo o modelo a testes, como comparar os resultados com eventos observados na realidade) (Eddy et al., 2012). A diretriz da ISPOR preconiza que a validação do modelo de uma AIO deve incluir pelo menos a validade aparente (validade de face) com os tomadores de decisão e a verificação dos cálculos (validade interna). A ISPOR recomenda ainda que, após a introdução da nova tecnologia, os dados sejam coletados e comparados com as estimativas da AIO, mesmo não sendo relevantes para a decisão já tomada; se os resultados estiverem próximos, isso proporcionará confiança na abordagem para futuras intervenções ou um aperfeiçoamento nos métodos. Mauskopf et al. (2017) apresentam métodos para três tipos de validação - validade aparente, validade interna e validade externa - e considera os três igualmente importantes e que devem ser incluídos em todas as análises de impacto orçamentário.

\section{Validade aparente (ou de face)}

A validade aparente pode ser estabelecida por revisão da estrutura do modelo, pressupostos, valores de parâmetros de entrada-padrão e resultados, tanto por especialistas como 
pelos detentores de orçamento que são suscetíveis de utilizar os resultados da análise do impacto orçamentário. Essa revisão pode ser feita por meio de entrevistas individuais ou convocando um painel de especialistas para revisar e discutir a estrutura do modelo, os pressupostos e os insumos. É importante ter uma avaliação antes e depois da programação do modelo. A avaliação antes de os resultados estarem disponíveis assegura que as reações aos componentes do modelo pelos especialistas ou os detentores de orçamento não serão influenciados pelos resultados. Também permite o desenvolvimento de uma análise que captura os componentes considerados de importância para esses tomadores de decisão.

\section{Validade interna}

Trata-se de verificar se o modelo está correto e funcionando da forma planejada. É de primordial relevância para a credibilidade do modelo desenvolvido. É uma maneira de assegurar que as estimativas de custos, as entradas de parâmetros (inputs) e os cálculos estão corretos. Mauskopf et al. (2017) sugerem uma lista de verificação para o modelo programado para execução da AlO: o texto está claro e conciso, sem erros ortográficos; todos os dados de entrada estão descritos e extraídos corretamente de suas respectivas fontes; há revisão e transparência dos cálculos; no caso de utilização de planilhas para cálculos, verificar se as programações e simulações estão corretas, funcionando adequadamente e se estão documentadas; se os resultados do modelo estão na direção esperada (para mais ou para menos), em função das entradas. Ainda se deve realizar verificações ao longo do processo de desenvolvimento e por pessoas diferentes que trabalham na análise. Também pode ser útil ter essas mesmas verificações realizadas por membros de outros projetos experientes de AIO, para garantir que as instruções para o usuário estejam claras.

É importante documentar quais revisões foram realizadas e descrever o que foi verificado e qual foi o resultado, quando aplicável. Por exemplo, ao testar se os cálculos do modelo funcionam como esperado ao mudar cada parâmetro um de cada vez, deve-se registrar em uma tabela cada entrada que foi alterada e testada, os resultados que ocorreram e se o resultado era esperado. Essa documentação deverá ser entregue como anexo ao modelo ou, ainda, apresentada na forma digital.

\section{Validade externa}

Estabelecer a validade externa de uma AlO é um processo projetado para verificar se os resultados da análise provavelmente serão precisos. Consiste em rodar seu modelo com dados reais. Ela pode ser estabelecida por meio da comparação das estimativas de análise de custos atuais ou impacto orçamentário com resultados relatados por outros modelos ou por observação retrospectiva ou prospectiva. Essa validação é importante para demonstrar a precisão do modelo para o tomador de decisão. Para uma AlO, o tipo mais simples de validade externa seria comparar as estimativas dos custos do ano corrente para a população elegível num cenário com a inserção da nova tecnologia, com dados oriundos de outras perspectivas (por exemplo, secretarias de saúde, saúde suplementar) (Mauskopf et al., 2017). A validação do modelo pode fornecer informações aos grupos elaboradores que permitam aperfeiçoar os métodos e fontes utilizadas para a modelagem.

\section{Análise de sensibilidade}

Consiste em analisar as incertezas do modelo proposto em função das estimativas obtidas. Possibilita descrever as fontes de incerteza dentro da $\mathrm{AlO}$ e demonstrar o impacto dessa incerteza sobre os resultados. Nessa análise, testa-se o impacto de diferentes pressupostos e valores dos parâmetros no resultado encontrado (Mauskopf et al., 2017).

Em geral, existem duas fontes primárias de incerteza associadas a qualquer AIO. A primeira fonte de incerteza são os valores de parâmetros e pressupostos (incluem características do paciente, incidência e prevalência da condição de saúde, custos atuais, padrão de tratamento e orientações de prática clínica). A segunda fonte de incerteza é para valores de parâmetros e outras suposições que são estimados com incerteza (por exemplo, real efetividade e segurança da tecnologia, market share, mudança de custos ao longo do horizonte de análise, mudanças no tratamento e outros eventos futuros) (Mauskopf et al., 2017).

Nessa análise são modificados os valores de estimativas e pressupostos com dados reais que não foram aplicados no modelo apresentado (por exemplo, população-alvo, distribuição por sexo e idade, dados de incidência e prevalência, cenários de tratamento, custos de aquisição da tecnologia, restrições de uso e custos de outros recursos). Os valores de entrada alternativos que impactam nos resultados podem ser apresentados em formato de tabela ou ilustrados usando um diagrama de tornado, que normalmente é utilizado para apresentar análise de sensibilidade univariada. Esse é o método recomendado na orientação do NICE sobre a estimativa do impacto financeiro e nas diretrizes atualizadas da Força-Tarefa de Impacto do Orçamento Econômico da Sociedade Internacional de Farmacoeconomia e Pesquisa de Desfechos (ISPOR). Na medida do possível, os intervalos ou valores alternativos utilizados para essas análises de sensibilidade devem basear-se na variabilidade observada em cada elemento de dados, e os métodos utilizados para derivar esses intervalos devem ser fornecidos. Por exemplo, os intervalos utilizados para a eficácia podem ser os intervalos de confiança de 95\% dos dados do ensaio clínico. No entanto, para muitas das variáveis incertas, não há dados observados. Portanto, a opinião de especialistas em intervalos prováveis pode ser necessária para análises de sensibilidade univariada. Em virtude da diferente 
variabilidade nos valores dos parâmetros, intervalos como \pm $20 \%$ para todos não devem ser rotineiramente utilizados. Para todos os valores alternativos testados nas análises de sensibilidade ou cenário, é necessário fornecer a fonte de dados e a lógica dos valores alternativos testados (Mauskopf et al., 2017).

\section{Software e interface de computador}

A plataforma mais utilizada é o Excel, com amplo e fácil acesso, familiar ao usuário, e permite o desenvolvimento de modelos flexíveis e interativos assegurando transparência e credibilidade. A partir das recomendações aqui apresentadas, foi desenvolvida uma planilha no Excel para execução da AIO. O manual com orientações de uso e a planilha foram disponibilizados gratuitamente no sítio: http://natsinc.org/wpress/ euroqol/.

\section{Outras considerações}

Por fim, como a revisão da literatura nos evidencia, há uma tendência das $\mathrm{AlO}$ de ficarem com frequência restritas à publicação na literatura cinzenta. As Diretrizes Metodológicas de AIO brasileiras devem traçar estratégias que estimulem a publicação e a disponibilização desse tipo de estudo econômico em bases de dados de amplo acesso, bem como posterior validação do seu modelo após a incorporação de determinada tecnologia. Essa validação pode representar uma valiosa fonte de dados para o a definição das melhores práticas de metodologia em AIO e sua consolidação no processo de tomada de decisão em saúde.

\section{Conclusão}

Avaliação de Impacto Orçamentário é uma ferramenta feita para auxiliar o gestor. A AlO prima por tentar garantir um equilíbrio fiscal ao sistema diante das inúmeras demandas da sociedade. Essa característica é fundamental no mundo atual, onde transparência e responsabilidade nos gastos são exigências cada vez mais frequentes por parte do cidadão. É importante salientar que uma AlO não visa produzir estimativas exatas da incorporação de novas tecnologias, e sim confeccionar um estudo sobre o tema a partir dos melhores dados possíveis. Além das avaliações econômicas, certamente outras variáveis serão fundamentais para a tomada de decisão em saúde, como relevância política, equidade, aspectos éticos e crenças pessoais. Prover uma excelência metodológica para avaliações econômicas baseadas em evidências científicas certamente contribuirá para melhor alocação de recursos e decisões para a sociedade.

\section{Referências bibliográficas}

Boulenger S, Ulmann P. French guidelines for the economic evaluation of health care technologies. Paris: College des Economistes de la Sante; 2004
Brasil. Agência Nacional de Vigilância Sanitária - Anvisa. Câmara de Regulação do Mercado de Medicamentos - CMED. Compras Públicas. Disponível em: http://portal.anvisa.gov.br/cmed. Acesso em: 3 abr. 2019

Brasil. Lei no 12.401, de 28 de abril de 2011, $190^{\circ}$ da Independência e $123^{\circ}$ da República. Altera a Lei no 8.080, de 19 de setembro de 1990, para dispor sobre a assistência terapêutica e a incorporação de tecnologia em saúde no âmbito do Sistema Único de Saúde - SUS. Diário Oficial da União 29 abr. 2011; Seção l:1 (publicação original).

Brasil. Ministério da Saúde. Departamento de Informática do SUS. Sistema de Gerenciamento da Tabela de Procedimentos, Medicamentos e Órteses, Próteses e Materiais Especiais do SUS - SIGTAP. Disponível em: http:// sigtap.datasus.gov.br/tabela-unificada/app/sec/inicio.jsp. Acesso em: 3 abr. 2019.

Brasil. Ministério da Saúde. Secretaria de Ciência, Tecnologia e Insumos Estratégicos. Departamento de Ciência e Tecnologia. Diretrizes metodológicas: análises de impacto orçamentário: manual para o Sistema de Saúde do Brasil. Brasília: Ministério da Saúde; 2012. 76p.

Brasil. Ministério da Saúde. Secretaria de Ciência, Tecnologia e Insumos Estratégicos. Portaria n² 26, de 12 de junho de 2015. Aprova os requisitos para submissão e análise de proposta de incorporação, alteração ou exclusão de tecnologia em saúde no SUS, por iniciativa do Ministério da Saúde e de Secretarias de Saúde dos Estados, dos Municípios e do Distrito Federal. Disponível em: http://conitec.gov.br/. Acesso em: 3 abr. 2019.

Brasil. Ministério da Saúde. Secretaria-Executiva. Departamento de Economia da Saúde, Investimentos e Desenvolvimento. Coordenação Geral de Economia da Saúde. Banco de Preços em Saúde. s/d. Disponível em: http://bps.saude.gov.br/. Acesso em: 3 abr. 2019.

Brasil. Portal de Compras do Governo Federal - Compras Governamentais. s/d. Disponível em: https://www.comprasgovernamentais.gov.br/index. php/consultass. Acesso em: 3 abr. 2019.

Cohen JP, Stolk E, Niezen M. Role of budget impact in drug reimbursement decisions. J Health Polit Policy Law. 2008;33(2):225-47.

Eddy DM, Hollingworth W, Caro JJ, Tsevat J, McDonald KM, Wong JB. Model transparency and validation: a report of the ISPOR-SMDM modeling good research practices task force - 7. Value Health. 2012;15(6): 843-50.

Garay OU, Caporale JE, Pichón-Rivere A, Marti SG, Mullen MM, Augustovski F. El analisis de impacto presupuestario en salud: puesta al dia con un modelo de abordaje genérico. Rev Peru Med Exp Salud Publica. 2011;28(3):540-7.

Garattini L, van de Vooren K. Budget impact analysis in economic evaluation: a proposal for a clearer definition. Eur J Health Econ. 2011;12(6):499-502.

Husereau D, Drummond M, Petrous S, Carswell C, Moher D, Greenberg D, et al. Consolidated Health Economic Evaluation Reporting Standards (CHEERS) statement. Value Health. 2013;16(2):e1-5.

Ireland. Guidelines for the Budget impact analysis of health technologies in Ireland. In: Authority HlaQ, editor. 2010. Disponível em: www.hiqa.ie. Acesso em: 3 abr. 2019.

Malaysia. Pharmacoeconomic Guideline for Malaysia. In: Malaysia MoHo, editor. 2012

Marshall DA, Douglas PR, Drummond MF, Torrance GW, Macleod S, Manti O, et al. Guidelines for conducting pharmaceutical budget impact analyses for submission to public drug plans in Canada. Pharmacoeconomics. 2008;26(6):477-95

Mauskopf J, Earnshaw SR, Brogan S, Wolowacz S, Brodtkorb TH. Budgetimpact analysis of health care interventions - A practical guide. Adis; 2017. v. XVIII, 224p.

Moher D, Liberati A, Tetzlaff J, Altman DG; The PRISMA Group. Preferred reporting items for systematic reviews and meta-analyses: the PRISMA statement. PLoS Med. 2009;6(7):e1000097. 
NICE - National Institute for Health and Clinical Excellence. Assessing cost impact (methods guide). In: Excellence NIfHaC, editor. 2011. Disponível em: https://www.nice.org.uk/media/default/about/what-we-do/intopractice/costing_manual_update_050811.pdf. Acesso em: 3 abr. 2019.

Neumann PJ. Budget impact analyses get some respect. Value Health. 2007;10(5):324-5

Niezen MG, de Bont A, Busschbach JJ, Cohen JP, Stolk EA. Finding legitimacy for the role of budget impact in drug reimbursement decisions. International Journal of Technology Assessment in Health Care. 2009;25(01):49-55.

Orlewska E, Gulácsi L. Budget-impact analyses. Pharmacoeconomics. 2009;27(10):807-27.

Orlewska E, Mierzejewski P. Proposal of polish guidelines for conducting financial analysis and their comparison to existing guidance on budget impact in other countries. Value Health. 2004;7(1):1-10.

PBAC - Pharmaceutical Benefits Advisory Committee. Guidelines for the pharmaceutical industry on preparation of submissions to the Pharmaceutical Benefits Advisory Committee. Canberra, Australia: Pharmaceutical Benefits Advisory Committee. 2013.

Pharmacoeconomic Guidelines Around The World ISPOR Website.Disponível em: http://www.ispor.org/peguidelines/index.asp. Acesso em:
Poland. Agency for Health Technology ASssessment. Guidelines for conducting Health Technology Assessment (HTA). In: Assessment AfHT, editor. 2009.

Sullivan SD, Mauskopf JA, Augustovski F, Jaime Caro J, Lee KM, Minchin M, et al. Budget impact analysis-principles of good practice: report of the ISPOR 2012 Budget Impact Analysis Good Practice II Task Force. Value Health. 2014;17(1):5-14.

Sullivan SD. Selected jurisdiction-specific budget impact analysis guidelines and URL link. 2014;17(1):5-14.

The International Network of Agencies for Health Technology Assessment - INAHTA [homepage]. Disponível em: http://www.inahta.org/aboutinahta/. Acesso em: 3 abr. 2019.

Thiry N, Neyt M, Van De Sande S, Cleemput I. Belgian guidelines for economic evaluations. Int J Technol Assess Health Care. 2014;30(6):601-7.

Trueman MP, Drummond M, Hutton J. Developing guidance for budget impact analysis. Pharmacoeconomics. 2001;19(6):609-21.

van de Vooren K, Duranti S, Curto A, Garattini L. A critical systematic review of budget impact analyses on drugs in the EU countries. Appl Health Econ Health Policy. 2014;12(1):33-40.

Watkins JB, Danielson D. Improving the usefulness of budget impact analyses: a US payer perspective. Value Health. 2014;1(17):3-4. 